\title{
Posterior Slope of the Tibia Plateau in Malaysian Patients Undergoing Total Knee Replacement
}

\author{
R Yoga, MS Ortho, N Sivapathasundaram*, MS Ortho, C Suresh**, MS Ortho \\ Department of Orthopaedics, Sultanah Aminah Hospital, Johor Bahru, Malaysia \\ *Department of Orthopaedics, Malacca Hospital, Malacca, Malaysia \\ **Department of Orthopaedics, Alor Star Hospital, Alor Star, Malaysia
}

\begin{abstract}
The posterior slope of the tibial plateau is an important feature to preserve during knee replacement. The correct slope aids in the amount of flexion and determines if the knee will be loose on flexion. This is a study on the posterior tibial plateau slope based on preoperative and postoperative radiographs of 100 consecutive patients who had total knee replacements. The average posterior slope of the tibia plateau was 10.1 degrees. There is a tendency for patients with higher pre-operative posterior tibial plateau slope to have higher post-operative posterior tibial plate slope.
\end{abstract}

Key Words:

Tibial plateau, Osteoarthritis, Arthroplasty, Posterior Slope, Knee Arthroplasty, Range of Motion

\section{INTRODUCTION}

One of the factors determining the outcome of total knee replacement (TKR) is proper placement of the prosthesis ${ }^{1}$; in fact, it is well known that there is a significant correlation between good clinical results and a well-positioned prosthesis ${ }^{2}$. The tibial component performs best when positioned horizontally in the lateral view ${ }^{3}$. This position however, does not correspond to the normal tibial anatomic slope. Whiteside ${ }^{4}$ suggested that the tibial cut should made at a $0^{\circ}$ to $3^{\circ}$ posterior slope, and some degree of posterior slope is now recommended when cutting the tibial plateau with most modern TKA designs.

We elected to study the posterior tibial plateau slope in Malaysian patients undergoing TKR and examine if there are any differences among the 3 major races of the Malaysian population. In addition, we wanted to know review the alignment of tibia plates following TKR in these patients.

\section{MATERIALS AND METHODS}

One hundred consecutive patients who underwent knee replacements were initially included for this study. These patients were treated in 2 major public hospitals, Sultanah
Aminah Hospital (50 patients) and Alor Star Hospital (50 patients). Patients who had large bone cysts, severe osteoporosis or marked deformity from rheumatoid arthritis were subsequently excluded from the study. Patients who did not have a true anterior-posterior and lateral postoperative radiographs were also excluded from this study. There were 3 different types of implants used : Apollo (Centerpulse), Nex Gen (Zimmer), Scorpio (Stryker).

The pre-operative and post-operative knee flexion and extension were recorded for each patient. Preoperative and immediate postoperative radiographs of all patients were reviewed. Firstly, a line was drawn parallel to the tibial plateau. A second line was then drawn centring on the middle of the tibia (anatomical axis) and intersecting the first line, simulating the intramedullary rod for the tibial cutting jig. The posterior slope calculated was $90^{\circ}$ minus the angle formed between the first line and the second line. In the immediate postoperative radiographs, a line was drawn parallel to the base of the tibial plate. The second line was then drawn centring on the tibial anatomical axis. Similar measurements were then taken. Horizontal or slight posterior slope of the tibia plate is desirable $(3,4)$. We identify patients with post-operative posterior tibial plateau slope (posterior slope of base plate) greater than 7 degrees for analysis.

The measurements were analysed using a statistical package (SPSS for Windows, Version 9.0). Significance was defined as a $\mathrm{p}<0.05$.

\section{RESULTS}

Nine patients were excluded for the study based on our selection criteria. Among the remaining 91 patients, there were 17 males and 74 females. The mean age of these patients was 62 years (range, $43-80$ years). Of these patients, $34 \%$ were Malays, $56 \%$ were Chinese and $10 \%$ were Indians. Fifty-six of the cases involved surgery on left knees and $44 \%$ on right knees.

The mean posterior tibial plateau slope of all the patients was 10.1 degrees (range 4- 21 degrees; $\mathrm{SD}=3.9$ degrees). 
Table I: The posterior tibial plateau slope distribution among the three different races

\begin{tabular}{|lccc|}
\hline Race & Mean Posterior Tibial Plateau Slope (degrees) & Range of knee motion (degrees) & Standard Deviation \\
\hline Malay & 10.0 & $4-21$ & 3.8 \\
Chinese & 9.9 & $4-20$ & 4.2 \\
Indian & 8.8 & $4-21$ & 3.7 \\
\hline
\end{tabular}

Table II: : Comparison between patients with higher or lower post-operative posterior tibial slope

\begin{tabular}{|lcc|} 
& \multicolumn{2}{c|}{ Post operative posterior tibial slope } \\
& More than $\mathbf{7}$ degrees & $\mathbf{7}$ degrees or less \\
\hline Number (percentage) & $15(16.5 \%)$ & $76(83.5 \%)$ \\
Pre Operative Slope & 12.5 degrees & 9.6 degrees \\
Post Operative Slope & 9.2 degrees & 2.8 degrees \\
\hline
\end{tabular}

There was no significant difference in the posterior tibial plateau slope in the three races studied (Table I). There was also no statistically significant difference in the angle of the slope between the males and the females.

The mean post-operative tibial base plate slope was 3.8 degrees (range, 0-14 degrees; $\mathrm{SD}=3.3$ degrees) (Table II). The mean reduction in the postoperative slope was 6.6 degrees. ( $\mathrm{SD}=4.5$ degrees). There were four cases in which the slope increased by an average of 1.2 degrees. In patients for whom the posterior tibial plateau slope was reduced after surgery, the mean pre-operative fixed flexion deformity was 2.9 degrees ( $\mathrm{SD}=6.7$ degrees), and the mean range of knee motion was 105.9 degrees ( $\mathrm{SD}=15.7$ degrees). In comparison, those who had an increased posterior tibial plateau slope after surgery, they had a mean pre-operative fixed flexion deformity of 7.8 degrees ( $\mathrm{SD}=9.7$ degrees) and mean range of knee motion of 97.5 degrees $(\mathrm{SD}=15$ degrees). Fifteen of the patients had a post-operative tibial baseplate slope of more than seven degrees, which was considered to be not ideal.

The left knee was more likely to be involved in fixed flexion deformity than the right knee $(\mathrm{P}<0.05)$, and patients undergoing left knee surgery also had a higher pre-operative tibial plateau slope.

\section{DISCUSSION}

The normal values for the posterior tibial plateau slope are not well defined. Studies note that the normal tibial plateau is inclined posteriorly between zero and 15 degrees ${ }^{5,6}$. A value of five to ten degrees is generally considered to be normal normal. There is a three degree variation between the medial and lateral tibial plateau slope ${ }^{7}$. It is however more useful to have the values of the posterior tibial plateau slope in the degenerated knees. In this study, the mean preoperative posterior tibial plateau slope was 10.0 degrees. $\mathrm{Chiu}^{7}$ noted that the average posterior plateau slope in Chinese patients was 11.5 degrees, a result comparable to the 10.1 degrees noted for Chinese patients in this study. He also concluded that osteoarthritis increases the slope by two to three degrees ${ }^{7}$. Although there are slight differences in mean pre-operative posterior tibial plateau slope between the 3 races, they are not statistically significant. It is likely that the study population was not big enough to elucidate the findings.

This study also noted that patients with higher pre-operative posterior tibial plateau slope are more likely to have higher post operative posterior tibial slope ${ }^{4}$. It is likely that knees with a higher pre-operative posterior tibial slope usually have fixed flexion deformity and reduced range of knee motion. In a study by Hoffman ${ }^{10}$, it was found that tibiae cut parallel to the surface exhibited $40 \%$ greater load carrying capacity and $70 \%$ greater stiffness than paired tibiae cut perpendicular to the long axis. Therefore, an increased tibial slope is not desirable although it improves the maximum flexion achieved ${ }^{11}$. Additional proximal tibia bone cut during surgery to achieve a lower posterior base plate slope may help to achieve full extension and better range of knee motion after surgery.

However, it has its own limitations. For example, if the posterior tibial slope is 15 degrees, and the anterior-posterior diameter of the proximal tibia is $50 \mathrm{~cm}$ wide, a zero degree tibia cutting guide will require the resection of $12 \mathrm{~mm}$ of bone from the anterior tibia. This removal exposes the weaker bone and predisposes the fixation to loosening. Observations by Bartel et al 8 suggested that when the stiffest and strongest cortical bone is removed, the remaining weaker and less stiff cancellous bone stock often is inadequate to support the physiologic loads of the knee. On the other hand, anteriorly sloped tibial components led to a tendency to posterior micromotion and thus more wear ${ }^{3}$. Inaccurate flexion extension gap also contributes to stiffness of the knee after TKA 9 .

\section{CONCLUSION}

The mean slope of the posterior tibia plateau in our pateints was 10.1 degrees and the values were not significantly different between the 3 major races in this country. Patients with higher pre-operative posterior tibial plateau slope are more likely to have higher post operative posterior tibial slope, which may adversely influence the knee extension and total range of knee motion. 


\section{REFERENCES}

1. Lotke PA, Ecker ML. Influence of positioning of prosthesis in total knee replacement. J Bone Joint Surg 1977; 59-A(1): 77-9.

2. Martin A, von Strempel A. Two-year outcomes of computed tomography-based and computed tomography free navigation for total knee arthroplasties. Clin Orthop Relat Res 2006; 449: 275-82.

3. Bai B, Baez J, Testa N, Kummer FJ. Effect of posterior cut angle on tibial component loading. J Arthroplasty 2000; 15(7): $916-20$.

4. Whiteside, LA, Amador DD. The effect of posterior tibial slope on knee stability after Ortholoc total knee arthroplasty. $J$ Arthroplasty 1988; 3 Suppl: S51-7.

5. Jiang CC, Yip, Liu TK. Posterior slope angle of the medial tibial plateau. J Formos Med Assoc 1994; 93(6): 509-12.

6. Matsuda S, Miura H, Nagamine R, Urabe K, Ikenoue T, Okazaki K, et al. Posterior tibial slope in the normal and varus knee. Am J Knee Surg 1999; 12(3): 165-8.

7. Chiu KY, Zhang SD, Zhang GH. Posterior slope of tibial plateau in Chinese. J Arthroplasty 2000; 15(2): 224-7.

8. Bartel DL, Burstein AH, Santavicca EA, Insall JN. Performance of the tibial component in total knee replacement. J Bone Joint Surg 1982; 64-A(7): 1026-33.

9. Bong MR, Di Cesare PE. Stiffness after total knee arthroplasty. J Am Acad Orthop Surg 2004; 12(3): 164-71.

10. Hofmann AA, Bachus KN, Wyatt RW. Effect of the tibial cut on subsidence following total knee arthroplasty. Clin Orthop Relat Res 1991; 269: 63-9.

11. Bellemans J, Robijns F, Duerinckx J, Banks S, Vandenneucker H. The influence of tibial slope on maximal flexion after total knee arthroplasty. Knee Surg Sports Traumatol Arthrosc.2005; 13(3): 193-6. 